\title{
Videokonferenzunterstützte Vorlesung - ein neues Element in der Ausbildung und eine Möglichkeit zur Verkehrsvermeidung
}

\author{
Prof. Dr.-Ing. habil. Bernd Hentschel, Dipl.-Ing. Peter Wasser
}

\section{Einleifung}

In Deutschland werden von Wirtschaft und Verwaltung jährlich etwa 62 Milliarden Deutsche Mark fuir Geschäftsreisen ausgegeben. Auch das damit verbundene Verkehrsaufkommen und die gleichzeitige Umweltverschmutzung sind nicht ohne Bedeutung. Durch den effektiven Einsatz von Videokonferenzen erschließt sich eine Möglichkeit diesen Aufwand deutlich zu reduzieren. Nach Berechnungen des Fernmeldetechnischen Zentralamtes in Darmstadt könnte das Verkehrsaufkommen um ca. $22 \%$ gesenkt werden, das entspricht etwa 7-15 Milliarden Personenkilometern im Jahr.

Bereits von 1975 bis 1982 wurden Anwendungsmöglichkeiten der Bildkommunikation in postinternen Netzen mit relativ hohem Kostenaufwand erprobt. Dabei war es bis zu vier Teilnehmern auf beiden Seiten möglich, über verschiedene Endgeräte Bildsprechfernsehen und auch Videokonferenzen abzuhalten.

Auf der Funkausstellung 1983 präsentierte die Deutsche Bundespost neue Lösungskonzepte fuir Videokonferenzen. Hierbei wurde der Informationsgehalt von Bewegtbildern durch ein bestimmtes Codier/Decodiergerät bereits auf bis zu $2 \mathrm{Mbit} / \mathrm{s}$ (entspricht etwa 30 digitalen Fernsprechkanälen) reduziert. 1984 wurden versuchsweise 13 öffentliche Videokonferenzräume eingerichtet. Ziel war es, die Bilder in Farbfernsehqualität zu übertragen. Dies war aber allein über Fernsprechkanäle nicht möglich. Durch die Entwicklung des BreitbandGlasfasernetzes, welches bereits seit 1983 bei dem Systemversuch BIGFON getestet wurde, ergaben sich neue und bessere Möglichkeiten für die Übertragung von Videokonferenzen. 1986 begann der Ausbau von Glasfaser-Overlaynetzen in 29 Städten. Ende 1988 waren bereits 188 Videokonferenzstudios angeschlossen. Nicht nur private Firmen, sondern auch Universitäten, die Post intern und staatliche Bereiche nutzten diese Studios. Am 23. Februar 1989 wurde das „VorläuferBreitbandnetz" in Betrieb genommen, welches ausgebaut und modernisiert zum ISDN- Netz wurde. Damit und mit der Entwicklung von Videokonferenzkarten für Personalcomputer wurde es möglich, Videokonferenzen mit einem tragbarem finanziellen Aufwand durchzuführen.

In diesem Zusammenhang entstand der Gedanke, an der Technischen Fachhochschule Wildau - parallel zu anderen Bildungseinrichtungen in Deutschland - eine Vorlesung als videokonferenzorientierte Vorlesung zu entwickeln. Die Wahl fiel auf das Fach „Grundlagen der Logistik". Gerade in diesem Fach war es von größter Bedeutung mit dem Grundgedanken der Einfuihrung moderner Lehrformen auch den verkehrsvermeidenden Aspekt dieser Variante an die Studenten zu vermitteln.
Auf der „Ersten Deutsch-Polnischen Logistikkonferenz" im März 1996 wurde das Euro-ISDN Netz erstmalig an der Technischen Fachhochschule Wildau für eine Videokonferenzschaltung genutzt. In Zusammenarbeit mit dem Fraunhofer Anwendungszentrum „Entsorgungsund Verkehrslogistik“ wurden in einer Demonstration die bereits vorhandenen Möglichkeiten den Zuhörern vorgestellt. Parallel dazu erfolgte die Einbeziehung einer studentischen Projektgruppe der Seminargruppen B1 und B2 / 95 bereits in die Vorbereitungsphase dieser Konferenz. Das Projekt „FACE to FACE“, an dem 5 Studenten mitwirkten, war einer der vorbereitenden Schritte für eine neue Form der Vorlesung an der Technischen Fachhochschule Wildau. In der Plenarsitzung wurde diese Variante sehr erfolgreich von den Studenten vorgetragen.

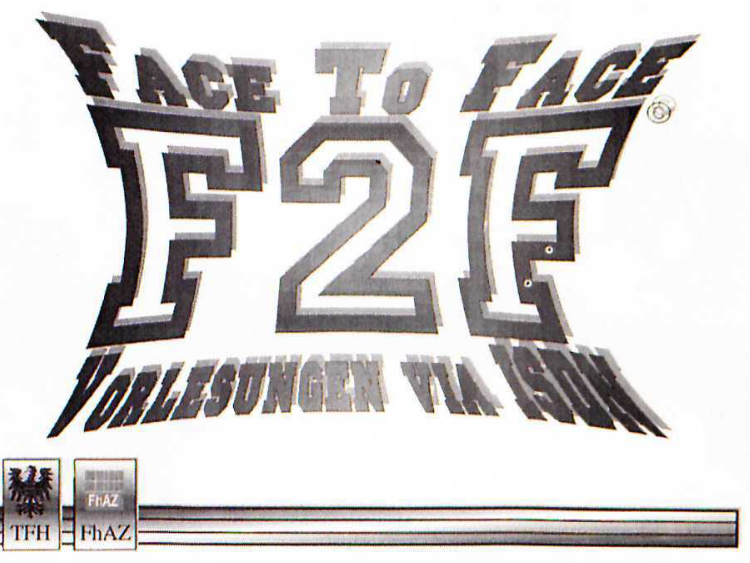

Bild 1 : „FACE to FACE“-Ankündigung

\section{Erste videokonferenzunterstützte Yorle- sungen im Wintersemester 1996/97}

Auf das Interesse der Studenten gestuitzt wurde im Wintersemester 96/97 im Fachgebiet Produktionslogistik mit der Einfuihrung videokonferenzunterstuitzter Vorlesungen begonnen. Erstes Vorlesungsfach war wie vorgesehen das Fach „Grundlagen der Logistik“. Das pädagogische Ziel war, eine Vorlesung so anzubieten, daß moderne praktische Lösungen in Logistikprozessen oder Logistikdienstleistungen simultan in Verbindung mit dem Projektleiter demonstriert werden und in anschließenden Wechselgesprächen zwischen Studenten und Projektleiter projektspezifische Fragen erörtert werden konnten.

Diese Form der Vorlesung ersetzt somit die ansonsten notwendigen Exkursionen mit aufwendigen Verkehrsplanungen und Kosten und ermöglicht eine vielfache praxisorientierte Demonstration moderner Lösungen. Damit ist zugleich ein Schritt in eine lebendige Vorle- 
sung getan. Was sonst nicht durch den Lehrenden in überzeugender Weise vorgetragen werden kann, ist somit durch die „Zuschaltung“ eines Projektleiters mit seinen unzähligen Projekterfahrungen möglich geworden. Außerdem kann in ïberzeugender Weise den Studenten verdeutlicht werden, wie mit dieser Methode zur Verkehrsvermeidung der logistische Slogan „Nicht Kilotonnen transportieren, sondern Kilobytes auf Reisen bringen !" zum Leben erweckt wird. Ein Beispiel einer Videokonferenzschaltung verdeutlicht Bild 2.

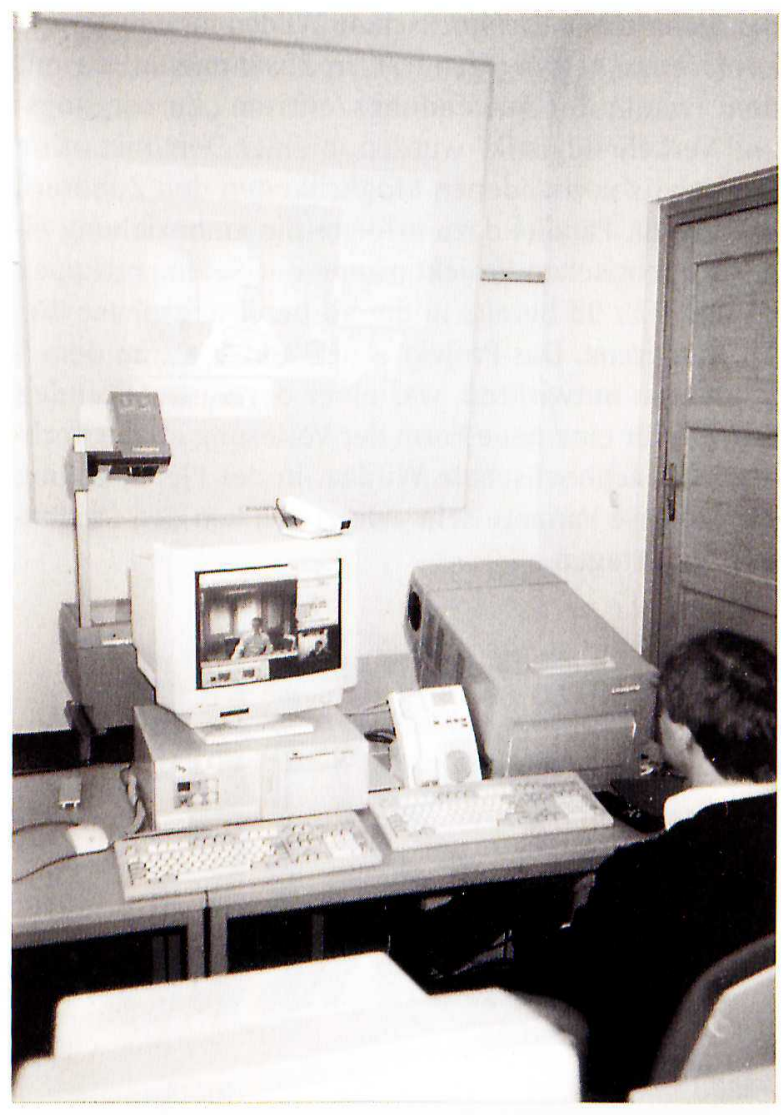

Bild 2 : Videokonferenzschaltung mit dem Fraunhofer Anwendungszentrum

Praxispartner, wie ein Anbieter automatisierter Parksysteme aus Süddeutschland, das Fraunhofer Anwendungszentrum „Entsorgungs- und Verkehrslogistik“ Prieros sowie das Mutterinstitut für Materialfluß und Logistik in Dortmund und die Projektlogistik GmbH Berlin konnten im Rahmen des Faches „Grundlagen der Logistik“ ihre Produkte präsentieren und die Studenten erhielten gleichzeitig die Möglichkeit, gezielt Fragen zur entsprechenden Problematik zu stellen. Die jeweiligen Partner wurden zu folgenden Themenkomplexen in Form einer 20- bis 30-minuitigen Liveschaltung in die Vorlesung integriert:

- Beispiele zur Bewältigung des ruhenden Verkehrs (Wöhr GmbH) - Vorstellung der automatisierten Parksysteme

- Beispiele zu entsorgungslogistischen Lösungen ( IML Dortmund, FHAZ Prieros)

- Füllstandsanzeige Wertstoffcontainer bzw. Demontagezelle für Fernsehgeräte

- Qualitätsicherung in der Logistik (Projektlogistik $\mathrm{GmbH})$ - Qualitätsmanagement
- Simulation in der Logistik (Projektlogistik GmbH) Bootssimulator bzw. Materialflußsimulation Finsterwalde

Das Beispiel Bootssimulator und seine Demonstration war sehr aufschlußreich. In Bild 3 ist der prinzipielle Aufbau des Simulators (steht heute in der TFH Wildau) zu sehen.

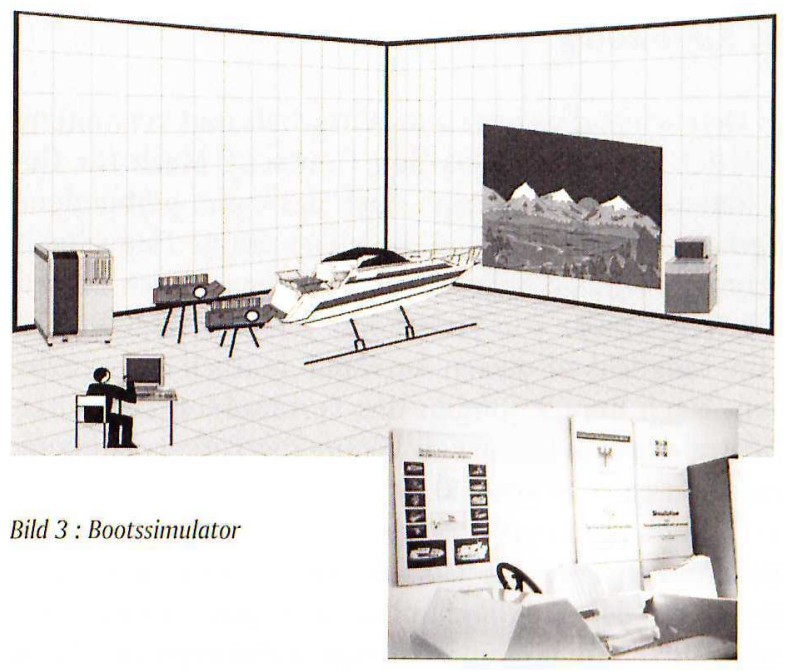

Zu allen interessierenden technischen Details und zur Umsetzung komplexer logistischer Lösungen waren die Entwickler entsprechender Anlagen bzw. Lösungen natürlich viel aussagefähiger. Diese ersten Videokonferenzen erbrachten Erfahrungen in positiver aber auch in negativer Hinsicht, auf die wir später noch näher eingehen möchten.

\section{Pädagogisch didaktisches Konzept und Gestaltung des Vorlesungsraumes}

Bevor wir mit dem Einbau von Videokonferenzen in den Vorlesungsbetrieb begonnen haben, war es notwendig, jedes Detail der Konferenz in Form eines exakten Ablaufplanes festzuhalten. Wir raten jedem davon ab, ohne ein pädagogisch-didaktisches Konzept videokonferenzunterstuitzte Vorlesungen durchzufuihren. Vorlesungen dieser Art erfordern einen erheblichen Aufwand an Vorbereitung, der sich aber durch die Wiederverwendbarkeit der einzelnen videokonferenzunterstuitzten Abschnitte sehr bald rentiert. Von großer Bedeutung ist das abgestimmte und vorher durchgesprochene inhaltliche Konzept mit dem jeweiligen Partner. Für die studentischen Zuhörer ergaben sich auch Anforderungen, die in Form einer disziplinierten Fragestellung gipfelten und kein „Dazwischenreden“ mehr zuließen. Bei videokonferenzorientierten Vorlesungen ist eine sehr große Disziplin zwischen Vortragendem und Zuhörer zu sichern. Der Gewöhnungsprozeß, den jeweiligen Partner erst ausreden zu lassen, war mühsam durchzusetzen. Immer wieder kamen sporadische Einwürfe und führten zu Blockaden beider beteiligter Seiten. Aber letztlich war es eine Frage der Zeit, bis die Studenten in einem disziplinierten Wechselgespräch die Vorteile dieser Vorlesungsform erkannten und stark begrüßten. 


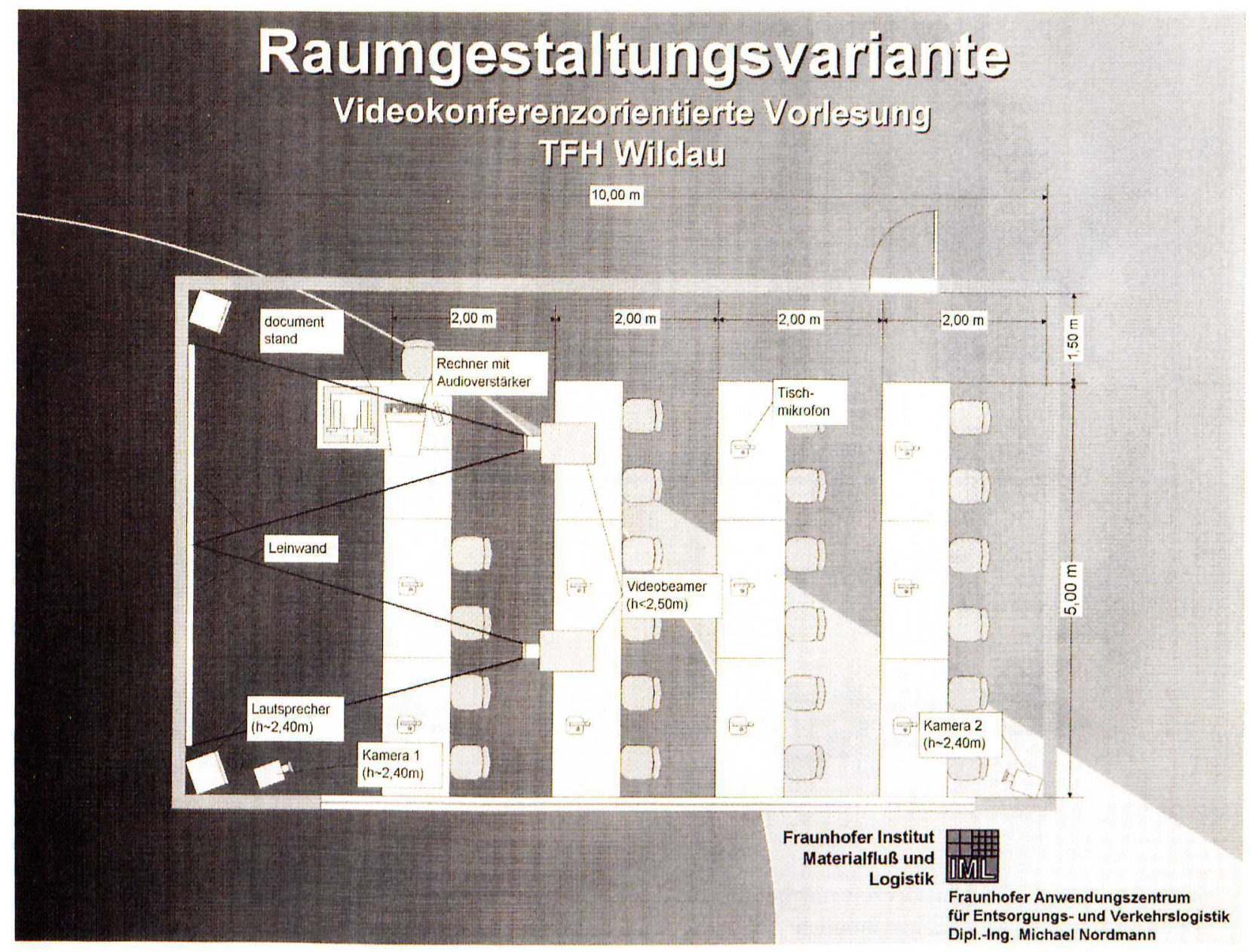

Bild 4: Raumgestaltungsvariante Videokonferenzorientierte Vorlesung

Ein weiterer Schwerpunkt für die Realisierung ist die technische Umsetzung und die Gestaltung des Vorlesungsraumes. In Bild 4 ist der Idealfall für einen Vorlesungsraum mit Videokonferenzkomponenten, den wir im vergangenen Semester noch nicht vollständig erreichen konnten, dargestellt. Folgende technische Ausrïstungen sind notwendig:

- Personalcomputer (ab Pentium 100 mit mind. 32 MB Hauptspeicher)

- Videokonferenzkarte (Standard H 320 und T 120) mit Kanalbuindelung

- bewegliche Kamera mit Zoom (2)

- mind. 1 Mikrofon je Vorlesungsplatz

- Daten- und Videoprojektor

- Videokonferenzsoftware mit Application sharing

- idealerweise 3 ISDN-Basisanschliisse mit 6 B-Kanälen

- entsprechend gekoppelte Audiotechnik

Bei der Zusammenstellung dieser Komponenten kommen uns die Erfahrungen aus dem Probebetrieb entgegen. Wir möchten natürlich auch die noch vorhandenen Probleme aufzeigen:

- Die an der Fachhochschule eingerichteten ISDNBasisanschluisse reichen für weitergehende Videokonferenzen (Bündelung von 6 Kanälen) nicht aus.

- Die teilweise Mehrfachnutzung von Anschlüssen führte zu Unterbrechungen in den Videokonferenzen und hinterließen bei unseren Praxispartnern und den Studenten keinen guten Eindruck.
- Die Qualität bei der Übertragung mit 2 B-Kanälen (128 kbit) entspricht bei den derzeitigen Kodierverfahren noch nicht den Ansprüchen der Studenten.

- Die erreichte Tonqualität führte nach längeren Videokonferenzen zu Ermuidungserscheinungen.

- Bei Applicationsharing ist bis jetzt keine Kompatibilität der verschiedenen Kartenhersteller zu verzeichnen.

- Zur Einbeziehung aller Studenten in die Videokonferenz ist eine sprachgesteuerte zoomfähige Kamera erforderlich.

Diese Probleme werden uns aber nicht davon abschrekken unseren Weg bei der Einbeziehung „Neuer Medien“ in den Vorlesungsbetrieb fortzusetzen. Denn Videokonferenzen haben erhebliche Vorteile:

- hohe Zeiteinsparungen

- hohe Flexibilität und Spontanität

- geringer Betriebskostenaufwand - keine Übernachtungs- und Anfahrtskosten; vergleichbar geringe Netzkosten

- weniger Fehler beim Informationsaustausch

- gemeinsame Arbeit an Dokumenten über große Entfernungen

Diese wiegen die durchaus vorhandenen Nachteile wie:

- Investitionskosten (befinden sich jedoch stetig im Abwärtstrend)

- indirekter persönlicher Kontakt mit den Gesprächspartnern 


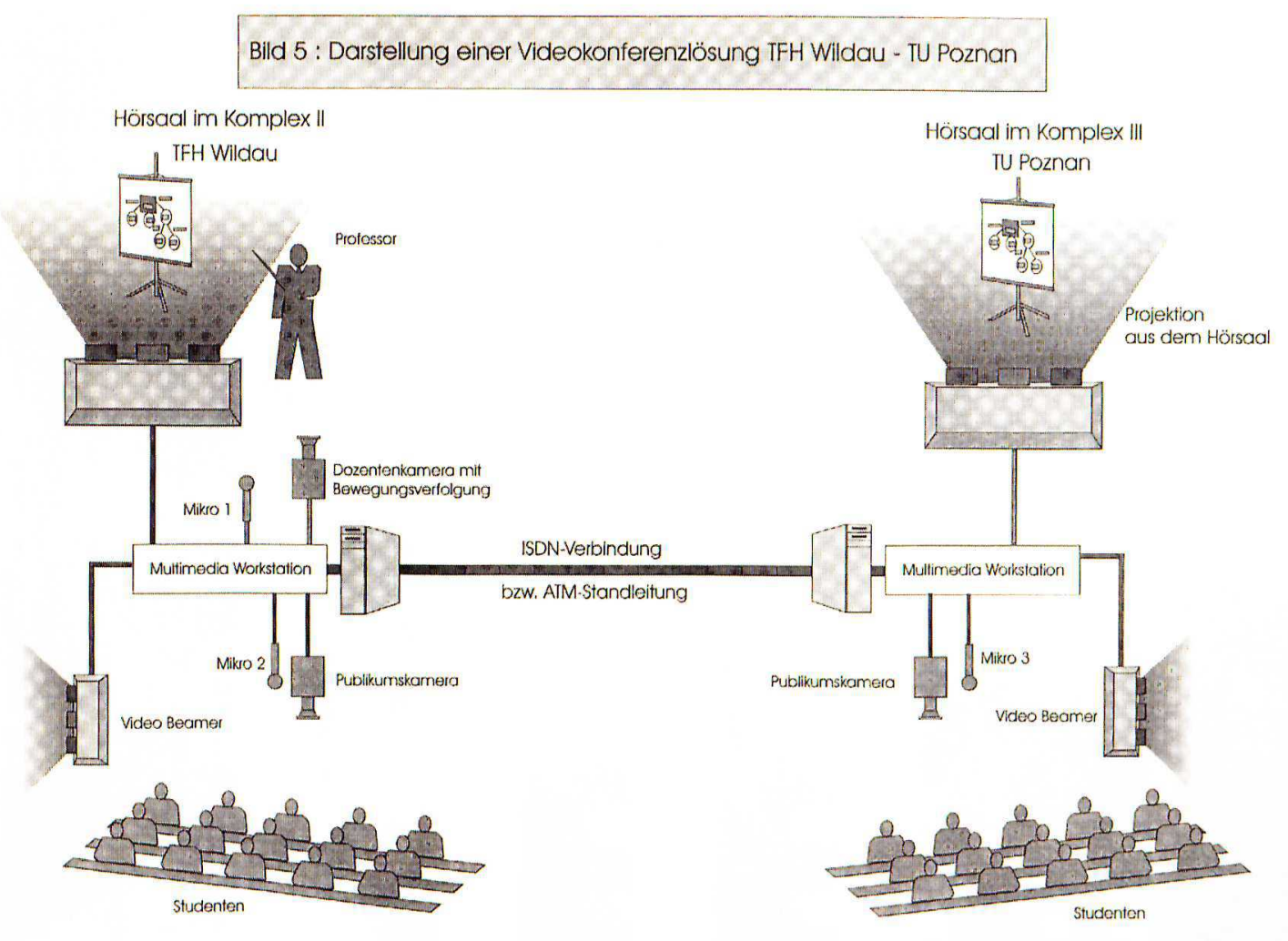

Bild 5 : Darstellung einer Videokonferenzlösung TU Poznan - TFH Wildau

- sinnvoller Einsatz (Kosten) derzeit nur bei bis zu 5 Konferenzpunkten gleichzeitig möglich bei weitem auf. Gleichzeitig können wir unsere Erfahrungen bei der Umsetzung in Industrieprojekte nutzen.

Dazu ein Beispiel der Zusammenarbeit mit mehreren mittelständigen Unternehmen unserer Region. Mit der Einfuihrung von videokonferenzunterstuitzten Schulungen, die in enger Zusammenarbeit und auf Grundlage unserer Erfahrungen mit einem ortsansässigen Unternehmen in Angriff genommen werden, entstehen Möglichkeiten, Partner von einem zentralen Standort aus an den unterschiedlichsten Orten zu unterweisen. Im Herbst 1997 werden wir eine Versuchsstrecke zwischen diesem Unternehmen, der Technischen Fachhochschule Wildau und dem Fraunhofer Anwendungszentrum auf Basis von Picture-Tel Videokonferenzkarten in Betrieb nehmen. Grundgedanke ist es, ein Präsentationszentrum fuir

- videokonferenzorientierte Beratungen als Dienstleistungszentrum in der Region

- Telewartungslösungen und zugehörige Beratungen

- studentisches Training im Beratungs- und Wartungsbereich

als Pilotlösung zu installieren und erste Erfahrungen zu sammeln. Gleichzeitig dient es als Kristallisationskern für weitere Entwicklungen.

\section{Ausblick}

Laut einer Prognose werden die Videokonferenzen über ISDN in etwa zehn Jahren das Telefon im Geschäftsbereich vollständig abgelöst haben. Auch im Aus- und
Weiterbildungsbereich werden die „Neuen Medien“ ihren Siegeszug fortsetzen. Auf der „Zweiten PolnischDeutschen Logistikkonferenz" werden wir unsere Kontakte dahingehend vertiefen, daß zukünftig ein Austausch von Vorlesungen über Videokonferenz auf internationaler Basis zwischen der Technischen Universität Poznan, dem Institut für Logistik Poznan und der Technischen Fachhochschule Wildau möglich sein wird. Dazu werden entsprechende Videokonferenzmodule in beiden Sprachen erarbeitet. Zukuinftig sollte auch fachbereichsübergreifend an der Ausstattung des großen Hörsaals mit Videokonferenztechnik gearbeitet werden, um ideale Bedingungen für Videokonferenzen zu erreichen. Unsere Bereitschaft zur Mitarbeit besteht.

Mit diesen ersten Ergebnissen und den geplanten Aktivitäten an der Technischen Fachhochschule Wildau sind wir sicher, daß unsere Fachhochschule im nationalen als auch internationalem Rahmen und insbesondere bei unseren Studenten weiter an Attraktivität gewinnt.

\section{Verfasser}

Prof. Dr.-Ing. habil. Bernd Hentschel

Dipl.-Ing. Peter Wasser

Technische Fachhochschule Wildau

Fachbereich Ingenieurwesen/Wirtschaftsingenieurwesen Fachgebiet Produktionslogistik

Tel.: (03375) 508927 